Complications travel: A cross-cultural comparison of the proportion of complications as a verbal cue to deceit

\author{
Aldert Vrij \\ Sharon Vrij
}

Author Note

Aldert Vrij, Sharon Vrij Department of Psychology, University of Portsmouth.

This work is funded by the High-Value Detainee Interrogation Group, DJF15-1299-V-0010271 awarded to the University of Portsmouth (UK). Any opinions, findings, conclusions, or recommendations expressed in this article are those of the authors and do not necessarily reflect the views of the U.S. Government.

Correspondence concerning this article should be addressed to Aldert Vrij, Department of Psychology, University of Portsmouth, King Henry Building, King Henry 1 Street, PO1 2DY, Hants, United Kingdom. Email: aldert.vrij@port.ac.uk 


\begin{abstract}
Verbal lie detection tools are almost exclusively examined in Western Europe and North America, but practitioners worldwide show interest in such tools. Since cultural differences in the efficacy of such tools may occur, it is important to examine their efficacy in different cultures. In this article, we focus on proportion of complications, which in previous research was more diagnostic than 'total details' in two British samples and in three samples that each included Hispanic, Russian, and South Korean participants. In the present article, we merged the three non-British samples and analysed the data by presenting them for each cultural group separately. Truth tellers disclosed the details of a trip away they had made, whereas liars fabricated such details. Proportion of complications discriminated truth tellers from liars in all three groups and was consistently a better indicator of veracity than total details. Implications of the findings are discussed.
\end{abstract}

Keywords: verbal cues to deception, within-subjects comparisons, liars' strategies, complications, proportion of complications, total details 


\section{Complications Travel: A cross-cultural comparison of the proportion of complications as a verbal cue to deceit}

Verbal lie detection tools are almost exclusively examined in Western Europe and North America, but practitioners frequently ask whether such tools are effective across cultures (Vrij, Leal, \& Fisher, 2018). Since cultural differences may influence the efficacy of such tools, it is needed to examine their efficacy in different cultures before their widespread use can be recommended. In this article, we examined one verbal lie detection approach, which focuses on the proportion of complications, amongst Hispanic, Russian and South Korean participants.

Amongst the verbal cues to deception that researchers have examined during the last 30 years, total details is one of the strongest indicators. It emerged as the strongest cue amongst the 19 cues investigated in a meta-analysis of Criteria-Based Content Analysis (CBCA) research, a frequently used verbal veracity assessment tool (Amado, Arce, Fariña, \& Vilarino, 2016). The typical finding is that truth tellers report more details than liars (Amado et al., 2016; DePaulo et al., 2003; Vrij, 2008). Two reasons are typically given for this veracity effect. First, liars may be unable to fabricate enough details that also sound plausible, a conclusion derived from the CBCA literature (Köhnken, 2004). Second, liars may be unwilling to provide many details out of fear that some of them will give leads to investigators that will reveal that they are lying (Nahari, Vrij, \& Fisher, 2014).

Recently, Vrij and colleagues suggested that total details does not make optimum use of the different strategies truth tellers and liars typically employ (Vrij et al., 2017, 2018a, b, 2019). They argued that both truth tellers and liars will report details to appear convincing, but that the type of detail they report will differ. They proposed to distinguish between one type of detail that truth tellers are more likely to 
report (complications) and two types of detail that liars are more likely to report (common knowledge details and self-handicapping strategies) and to use the proportion of cues to truthfulness (i.e. the proportion of complications) as a measure. They argued that since the proportion of complications takes the different strategies from truth tellers and liars better into account than total details, it should differentiate truth tellers from liars better than the total details variable.

A complication is an occurrence that makes a situation more difficult to report than necessary ("We did not see him, we were waiting at a different entrance") (Vrij et al., 2018b). Complications have been examined before and is one of the 19 criteria that constitutes the CBCA-list. According to a recent CBCA meta-analysis, complications are more likely to occur in truthful statements than in deceptive statements (Amado et al., 2016; Vrij, 2008; Vrij et al., 2017b). Truth tellers are thought to report more complications than liars because liars prefer to keep their stories simple (Hartwig, Granhag, \& Strömwall, 2007), whereas reporting complications makes a story more complex.

Common knowledge details refer to strongly invoked stereotypical information about events ("We visited the zoo and saw lots of animals") (Vrij et al., 2018b). Liars are more likely to include common knowledge details in their statements than truth tellers (Sporer, 2016; Volbert \& Steller, 2014), because when describing a made-up activity they lack the personal experiences truth tellers can report.

Self-handicapping strategies refer to justifications as to why someone is not able to provide information (Vrij et al., 2018b). Such justifications can be explicit ('I can’t remember his name, I am bad at remembering names") or implicit ("I slept in the train”). Liars are more likely to include self-handicapping strategies in their statements than truth tellers. For liars to keep their stories simple, not providing too 
many details is an attractive strategy. However, they run the risk not to be believed if they provide not enough detail. A solution is to offer a justification for not being able to report details.

In all five studies to date in which complications, common knowledge details, self-handicapping strategies and total details have been examined (Leal, Vrij, Deeb, \& Kamermans, 2019; Vrij et al., 2017, 2018a, b, 2019) ${ }^{1}$, it was found that truth tellers reported more complications and liars more self-handicapping strategies (liars did not always report more common knowledge details). In addition, in all five studies the proportion of complications was higher amongst truth tellers than liars. Also, the main hypothesis that the proportion of complications is more diagnostic in discriminating truth tellers from liars than total number of details was supported in all five studies.

In Leal et al. (2019) and Vrij et al. (2018b) the participants were British and the results have been fully reported. In each of the other three studies (Vrij, 2017, 2018a, 2019) participants were from Russian, South-Korean and Hispanic origin. Differences between these three nationalities (Russians, Koreans and Hispanics) were not discussed as nationality was always introduced as a covariate. Since all these three studies employed a similar procedure, it would be possible to merge these data sets of Vrij et al. (2017, 2018a, 2019). This has been done in the current article. The innovative aspect of the current article is the Group analyses in which we examined the verbal cues total details, complications, common knowledge details, selfhandicapping strategies and proportion of complications in each of the three nationalities. We thus addressed practitioners' request to focus on cross-cultural findings.

In the three studies that formed the basis of this article, participants were university students and interviewed by local interviewers in their own language at 
their local university. In theory we could make two comparisons: (i) Comparing the three groups for speech differences or (ii) comparing the difference between truth tellers and liars in each group. The first comparison is in the present sample somewhat problematic because the groups differed on various aspects, including being interviewed by different interviewers. Neither is this a comparison practitioners are particularly interested in. For them the crucial question is the cultural (in)stability of diagnostic cues to deceit, which is examined in the second comparison. The few studies that examined cross-cultural differences in verbal cues to deception (Leal et al., 2018; Taylor, Larner, Conchie, \& Menacere, 2017; Taylor, Larner, Conchie, \& van der Zee, 2017) found differences in the diagnostic value of these cues across cultures. This makes it worthwhile to examine differences in the diagnostic value of the verbal veracity cues total details, complications, common knowledge details, selfhandicapping strategies and the proportion of complications across cultures. We explored whether the main hypothesis that the proportion of complications is more diagnostic in discriminating truth tellers from liars than total number of details found support in all three Groups.

\section{Participants}

\section{Method}

A total of 608 University students (135 males, 466 females and seven unknown) took part in the three studies. Their age ranged from 18-42 years with an average age of $M=21.82$ years $(S D=3.41)$. The experiment took place in three different universities in Russia, South Korea and Texas, USA, and the participants were of Russian $(n=239)$, Korean $(n=240)$, and Hispanic $(n=129)$ origin.

Conducting a posthoc power analysis using $\mathrm{G}^{*}$ Power software revealed that for a one-tailed test, a moderate effect size of $\mathrm{r}=0.30$ (the equivalent of $d=0.586$, which is the average of $d$-scores for the dependent variables when the total sample is 
considered, see Table 1), an alpha error rate of 0.05 , and a total sample size of 608 , the power achieved is perfect (1.00). Also, for a sample of 129 (Hispanics group size), the power is 0.97 .

\section{Procedure}

The three studies on which this data set was based all used the same procedure. We will provide here a synopsis of the procedure and refer to the original articles by Vrij and colleagues for the full details (Vrij et al., 2017, 2018a, 2019). Participants were interviewed about an alleged trip to a city they made during the last twelve months. Truth tellers really had made the trip they discussed, whereas liars did not. In fact, they had never visited the city they talked about before. Both truth tellers and liars were given time to prepare themselves for the interview for which a computer with internet access was available. In a pre-interview questionnaire, participants rated their thoroughness of preparation via three items: (1) shallow to (7) thorough; (1) insufficient to (7) sufficient; and (1) poor to (7) good. The answers to the three questions were averaged (Cronbach's alpha $=.84$ ) and the variable is called 'preparation thoroughness'. Participants were also asked whether they thought they were given enough time to prepare themselves with the following question: 'Do you think the amount of time you were given to prepare was: (1) insufficient to (7) sufficient. Finally, they were asked how motivated they were to perform well during the interview: (1) not at all motivated to (5) very motivated.

The interview protocols used in the three studies differed somewhat, but all interview protocols invited interviewees to discuss their trip in as much detail as possible. Because of the differences between the interview protocols used in the three studies, we included 'study' as the first (of seven) covariates in the analysis (coded as a dummy variable). In the three studies, different manipulations took place to 
encourage interviewees to provide more details, such as the use of a model statement (Vrij et al., 2017) and the use of sketches (Vrij et al., 2018a, 2019). The effects of these manipulations have been discussed in the original articles by Vrij and colleagues and as such, will not be discussed here. With the obtained data sets, we introduced in the current analyses the presence of a manipulation (yes or no) as the second covariate (coded as a dummy variable). Finally, in the three studies the participants were interviewed either in their own native language by an interviewer who shares their native language or in their own native language by an Englishspeaking interviewer through an interpreter. The effects of the interpreter have also been discussed in the original articles and will not be discussed here. We included in the current analyses the presence of an interpreter (yes or no) as the third covariate (coded as a dummy variable).

In the post-questionnaire, which participants were asked to complete truthfully, participants were asked to indicate the extent to which they told the truth in the interview on a 11 point Likert scale ranging from $0 \%$ to $100 \%$. Rapport between the interviewee and interviewer was also measured via the nine items Interaction Questionnaire (Vallano \& Schreiber Compo, 2011). Participants rated the interviewer on 7-point scales ranging from [1] not at all to [7] extremely on nine characteristics such as smooth, bored, engrossed, and involved (Cronbach's alpha $=.84$ ).

\section{Coding}

The coders, all from the UK, were blind to the Veracity condition in the three studies. Some coders coded details, other coders coded complications, common knowledge details and self-handicapping strategies. A detail is in this study defined as a unit of information about the trip the interviewee allegedly had made. To give an example, the following answer has seven details: "Im also drawing a plaza that is in 
front of the cathedral. There was a large fountain. It had a lot of seats, or benches". Complications and common knowledge details are subcategories of details, but not all details are either complications or common knowledge details. In fact, the sentence above that contained seven details does not contain a complication or common knowledge detail. A complication is an occurrence that makes a situation more difficult to report than necessary. For example, the sentence "The hotel beach was full of little stones which made it painful to walk on" contains seven details and one complication (stones makes walking painful). Common knowledge details refer to strongly invoked stereotypical knowledge about events. The sentence "We also

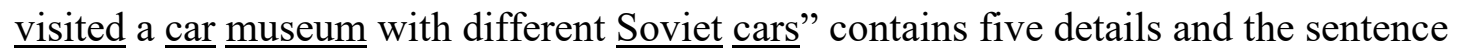
also counts as one common knowledge detail. Self-handicapping strategies refer to explicit or implicit justifications as to why someone is not able to provide information “I'm not very good at remembering names, so I will just tell you like common details" (Vrij et al., 2018b). For number of details, a second coder coded a selection of the transcripts independently from the first coder. For the remaining variables, two coders coded all transcripts independently from each other and discussed their disagreements. Reliability between the coders was measured via Intraclass correlation coefficients (ICC). Reliability in all three studies was good for coding details (ICC's ranged from .74 to .92) and complications (ICC's ranged from .74 to .92) and satisfactory for common knowledge details (ICC's ranged from .64 to .70) and selfhandicapping strategies (ICC's ranged from .68 to .85), see Vrij et al. (2017, 2018a, 2019). For all dependent variables, repetitions were never coded. We calculated the total number of details, complications, common knowledge details and selfhandicapping strategies reported in the entire interview. The proportion of 
complications was calculated as follows: complications/ (complications + common knowledge details + self-handicapping strategies).

\section{Results}

We present all statistical information for the Veracity main effect in Table 1 and for the Group main effect in Table 2. For the dependent variables presented in the Hypotheses-testing part of this Results section, we report the statistical information regardless of whether the Veracity or Group main effects were significant. For the remaining dependent variables, all presented before the Hypotheses-testing part, we only present the significant results in Tables 1 and 2.

\section{Preparation thoroughness, preparation time and motivation}

Insert Tables 1 and 2 about here

Three ANOVAs utilizing a 2 (Veracity: truth vs lie) X 3 (Group: Russians vs Koreans vs Hispanics) between-subjects design were carried out with preparation thoroughness, preparation time and motivation as dependent variables. For preparation thoroughness, a significant main effect for Veracity emerged, see Table 1. Truth tellers rated their preparation as more thorough than liars. The Group main effect was also significant, see Table 2. The Hispanic participants judged their preparation as more thorough than the Russians and Koreans. The Veracity x Group interaction effect was not significant, $F(1,602)=2.69, p=.069, \eta_{p}^{2}=.01$.

The Veracity main effect for interview time was also significant, see Table 1. Truth tellers believed more than liars that they were given sufficient time to prepare themselves for the interview. The Group main effect was also significant, see Table 2. Koreans judged their preparation time as significantly poorer than the Hispanics, who, in turn, judged it as poorer than the Russians. The Veracity x Group interaction effect was also significant, $F(1,602)=6.29, p=.002, \eta_{p}{ }^{2}=.02$. Simple effect tests showed 
that in all three countries truth tellers more than liars thought that they were given sufficient preparation time, but the effect sizes show that this difference was larger amongst Koreans (truth tellers $M=5.74, S D=1.38,95 \%$ CI [5.44, 6.04], liars $M=$ $4.30, S D=1.77,95 \%$ CI $[4.03,4.58]), F(1,238)=47.98, p<.001, d=.91(.63,1.16)$ than amongst Russians (truth tellers $M=6.43, S D=1.00,95 \%$ CI $[6.22,6.66]$, liars $M$ $=5.82, S D=1.38,95 \%$ CI $[5.60,6.04]), F(1,237)=15.47, p<.001, d=.50(.24, .75)$ and Hispanics (truth tellers $M=5.76, S D=1.42,95 \%$ CI [5.43, 6.09], liars $M=5.20$, $S D=1.48,95 \%$ CI $[4.82,5.59]), F(1,127)=4.65, p=.003, d=.39(.03, .73)$.

For motivation, The Veracity x Group interaction effect was significant, $F(1$, $602)=5.54, p=.004, \eta_{p}{ }^{2}=.02$, but the Veracity $F(1,602)=0.73, p=.392, \eta_{p}{ }^{2}=.001$ and Group $F(1,602)=1.05, p=.3502, \eta_{p}{ }^{2}=.003$ main effects were not. Simple effect tests showed that amongst Hispanic participants, truth tellers $(M=4.09, S D=$ $0.80,95 \%$ CI $[3.91,4.28])$ were more motivated than liars $(M=3.78, S D=0.84,95 \%$ CI $[3.56,4.00]), F(1,127)=4.63, p=.033, d=.38(.02, .73)$. Amongst the Koreans, liars $(M=4.00, S D=0.64,95 \% \mathrm{CI}[3.88,4.11])$ were more motivated than truth tellers (truth tellers $M=3.81, S D=0.72,95 \% \mathrm{CI}[3.68,3.94]), F(1,238)=4.66, p=$ $.032, d=.28(.02, .53)$, whereas amongst the Russians truth tellers $(M=4.01, S D=$ $0.64,95 \%$ CI $[3.89,4.12])$ and liars $(M=3.98, S D=0.61,95 \%$ CI $[3.87,4.10]), F(1$, $237)=0.10, p=.759, d=.05(-.21, .30)$ were equally motivated.

We introduced preparation thoroughness, preparation time and motivation (all interval variables) as covariates four to six in the Hypotheses-testing analyses.

\section{Rapport and percentage of truth telling}

Two ANOVAs utilizing a 2 (Veracity: truth vs lie) X 3 (Group: Russians vs Koreans vs Hispanics) between-subjects design were carried out with (1) rapport, and (2) percentage of truth telling as dependent variables. The analysis with Rapport 
resulted in a Group main effect, see Table 2. The Russians judged their rapport with the interviewer as significantly better than the Koreans and Hispanics. The Veracity main effect $F(1,602)=3.69, p=.055, \eta_{p}{ }^{2}=.006$, and Veracity x Group interaction effect, $F(1,602)=1.50, p=.223, \eta_{p}^{2}=.005$ were not significant.

The analysis with percentage of truth telling revealed a significant Veracity main effect, see Table 1. Truth tellers reported to have been more truthful than liars, showing that the manipulation was successful. The Group main effect $F(1,602)=$ 2.03, $p=.132, \eta_{p}{ }^{2}=.007$, and Veracity x Group interaction effect, $F(1,602)=2.07, p$ $=.127, \eta_{p}{ }^{2}=.007$ were not significant. We included Rapport (an interval variable) as the seventh covariate in the Hypotheses-testing analyses.

\section{Hypothesis-Testing}

Five ANCOVAs utilizing a 2 (Veracity: truth vs lie) X 3 (Group: Russians vs Koreans vs Hispanics) between-subjects design were carried out with the Number of details, Complications, Common knowledge details, Self-handicapping strategies and Proportion of complications as dependent variables. The seven covariates were study, experimental manipulation, interpreter preparation thoroughness, preparation time, motivation, and rapport. All five Veracity main effects and two of the five Group main effects were significant, see Tables 1 and 2. None of the Veracity x Group interaction effects were significant, all $F^{\prime} s<1.18$, all $p$ 's $>.308$.

Truth tellers included more total details and complications, and fewer common knowledge details and self-handicapping strategies into their accounts than liars. Also the proportion of complications was higher for truth tellers than for liars. The effect sizes show that total details resulted in the smallest effect $(d=.25)$ and proportion complications in the largest effect $(d=.94)$. According to Cohen's (1988) classification $^{2}$, total details resulted in a small effect, complications, common 
knowledge details and self-handicapping strategies in medium effects and the proportion of complications in a strong effect. Out of complications, common knowledge details and self-handicapping strategies, the strongest effect was found for complications. They also frequently occurred in truth tellers. Only seven out of 303 truth tellers $(2.3 \%)$ did not report a complication. We also calculated the Bayes Factors using JASP software. ${ }^{3}$ The Bayes Factor analyses revealed substantial to very strong evidence for all five effects.

Koreans included more complications in their reports than Russians, whereas Hispanics did not differ from either group, see Table 2. In addition, Koreans included more common knowledge details in their accounts than Russians and Hispanics, whereas the two latter groups did not differ from each other.

Despite the absence of any Veracity x Group interaction effect, we also report the results for the three groups separately in Table 1 because the aim of the current article was to analyse the results for the three groups separately. In all groups, a similar pattern of results emerged in that truth tellers reported more complications than liars, and liars more common knowledge details and self-handicapping strategies than truth tellers (although for Hispanics the common knowledge effect was not significant). The proportion of complications was always higher for truth tellers than liars.

For the Russians, total details resulted in a small effect, complications, common knowledge details and self-handicapping strategies in medium effects and the proportion of complications in a strong effect. However, the main effect for total details was not significant $(p=.052)$. The Bayes Factor analyses showed support for the null-hypotheses for total details and very strong evidence for the effects for the other variables in the Russian sample. 
For the Koreans, total details resulted in a small effect size, common knowledge details and self-handicapping strategies in medium effect sizes and complications and the proportion of complications in strong effect sizes. In Bayes Factors language, we found weak evidence for the effect for total details but very strong evidence for the remaining four variables.

For the Hispanics, we found for total details and common knowledge details small effect sizes and evidence for the null hypotheses and for the other variables medium effect sizes and very strong evidence for the alternative hypotheses. The hypothesis that the proportion of complications is a stronger indicator of deception than total details is supported, both in the total sample ( $d=.25 \mathrm{vs} d=.94)$ and for the three individual groups (total detail $d$-scores ranged from .22 to .36 and the proportion of complications $d$-scores ranged from .75 to 1.00).

We carried out six discriminant analyses to distinguish between truth tellers and liars in each of the three groups using either the number of reported details or the proportion of complications as predictors. The objective group belonging (truthful versus deceptive) was the classifying variable. Total details revealed a significant discriminant function only once, in the Korean sample see Table 3. The obtained accuracy rate was rather low, $54.20 \%$. The proportion of complications score resulted in significant discriminant functions in all three groups with total accuracy rates close to $70 \%$ in each of the three Groups.

Insert Table 3 about here

\section{Discussion}

The proportion of complications was significantly higher in truth tellers than liars in all three groups we examined and the effect sizes were strong in each group. This means that the proportion of complications shows stability across cultures, in 
other words, this cue 'does travel'. If we examine the three cues on which this proportion cue is based -complications, common knowledge details and selfhandicapping strategies- separately, it emerged that complications yielded the strongest effect and was significant in all three groups: Complications do thus travel as well. Common knowledge details did distinguish truth tellers from liars in the Russian and South-Korean samples but not in the Hispanic sample. The data do not provide us with the opportunity to explain the absence of an effect amongst Hispanic participants but may indicate that common knowledge details are not as diagnostic for veracity as complications and self-handicapping strategies are. The self-handicapping strategies scores discriminated truth tellers and liars in all three groups but did not occur often. This could be somewhat problematic in an applied setting because it means that the absence of the cue does not provide valuable information about the veracity status of the cue. In contrast, complications do occur frequently. Only seven out of 303 truth tellers $(2.3 \%)$ did not report a complication.

At this stage we can only speculate why proportion of complications and complications showed stability across cultures. These variables are based on truth tellers' and liars' strategies and perhaps such strategies do not differ much across groups. This is an empirical question worth examining.

The proportion of complications variable yielded accuracy rates close to $70 \%$, which is very similar to the accuracy rates obtained in two alternative, more established, verbal veracity tools: Criteria-Based Content Analysis (CBCA, Amado et al, 2016; Vrij, 2018; Vrij \& Fisher, 2016) and the Verifiability Approach (VA, Nahari, 2018; Vrij \& Fisher, 2016; Vrij \& Nahari, 2019). In sum, verbal veracity tools seem to result in accuracy rates of around $70 \%$; the next challenge for researchers is to improve on these rates. 
That the proportion of complications variable yields similar strong effects across countries is also good news for investigators. Lie detection is in itself a difficult task (e.g. Bond \& DePaulo, 2006) and it only further complicates things for investigators if some verbal cues are diagnostic in some groups but not in others. The latter happened when verbal cues such as person pronouns and spatial information were examined (Taylor, Larner, Conchie, \& Menacere, 2017; Taylor, Larner, Conchie, \& van der Zee, 2014).

Examining complications, common knowledge details, self-handicapping strategies and the proportion of complications yielded positive results in all five studies published to date (Leal, Vrij, Deeb, \& Kamermans, 2019; Vrij, 2017, 2018a, b, 2019), and, as the present article showed, revealed positive results across cultures. This may warrant recommendation to investigators to start examining these cues. We realise that it may be difficult for investigators in interviews to pay attention to all three cues in real time and it would be impossible for them to calculate the proportion score in real time. However, observing practitioners in training sessions revealed that they can count the number of complications reported in interviews in real time. This is encouraging news because complications is the strongest cue to veracity of the three cues.

The variable total details resulted in weaker effects than complications, common knowledge details and self-handicapping strategies. In fact, total details only resulted in a significant effect in the Korean sample, but even in that group it only showed weak evidence for the alternative hypothesis. The weak performance of the total details findings is not in alignment with the deception literature where it is typically found that total details is amongst the most diagnostic verbal cues to veracity. Our data set only allows us to speculate why this erratic result for total 
details emerged. One possibility is that this was caused by the opportunity we gave liars to plan their stories. Planning typically improves liars' performance (DePaulo et al., 2003; Sporer \& Schwandt, 2006, 2007) and perhaps even more in the deception scenario we used in which liars could easily embed their lies in truthful stories. That is, most liars probably have made trips in their life before and could thus have incorporated truthful details of such trips in their deceptive stories.

We believe that the weakness of the total details effect, rather than a cultural explanation, is the reason why a significant effect only emerged in the Korean sample. We cannot think of a theoretical explanation why the finding that truth tellers typically report more details than liars would depend on the culture, because we believe the reason why this is the case- liars are unable and unwilling to report as many details as liars- to be universal.

We believe that in the proportion of complications research domain three research activities in particular are welcome, all three related to testing the robustness of the effect. First, to date it has only been investigated in Vrij's lab, but replications of the findings across research labs will make the results more robust. Second, to date it only has been examined in one deception scenario, the travel scenario (Leal, Vrij, Deeb, \& Kamermans, 2019; Vrij et al., 2017, 2018a, b, 2019). Since the context is important for lie detection, research examining other scenarios would be welcome. Such scenarios need to be carefully chosen. It is important that complications, common knowledge details and self-handicapping strategies occur spontaneously, which means that the truths and lies participants report should be self-generated (Vrij et al., 2017). In most deception research truth tellers (and sometimes liars) are instructed to carry out relatively short specific tasks or to watch a video (Vrij, 2008). In such scenarios, the number of complications is then preliminary determined by the 
stimulus material. In studies where truth tellers and liars are allowed to choose their own activities they want to carry out -common procedure in the Verifiability Approach research (Nahari, 2018; Vrij \& Nahari, 2019), these activities are typically short of duration (around thirty minutes) which automatically limits the number of complications that could occur. Third, as noted by Nahari et al. (2019), the proportion of complications results would become stronger if truth tellers would report more complications and fewer common knowledge details. Researchers could design methods to encourage truth tellers to do this.

In conclusion, the results showed that complications, common knowledge details, self-handicapping strategies and proportion of complications are diagnostic cues to deceit in different cultures. We believe that these cues, particularly observing complications, may be ready for use by practitioners in real life. We recommend future research to focus on replication of the findings in different labs and different deception scenarios and on methods to encourage truth tellers to report more complications and fewer common knowledge details. 


\section{References}

Amado, B. G., Arce, R., Fariña, F., \& Vilarino, M. (2016). Criteria-Based Content Analysis (CBCA) reality criteria in adults: A meta-analytic review. International Journal of Clinical and Health Psychology, 16, 201-210. doi.org/10.1016/j.ijchp.2016.01.002.

Bond, C. F., \& DePaulo, B. M. (2006). Accuracy of deception judgements. Personality and Social Psychology Review, 10, 214-234. Doi:

$10.1207 /$ s15327957pspr1003_2

Cohen, J. (1988). Statistical power analysis for the behavioral sciences (2nd ed.). Hillsdale, NJ: Erlbaum.

DePaulo, B. M., Lindsay, J. L., Malone, B. E., Muhlenbruck, L., Charlton, K., \& Cooper, H. (2003). Cues to deception. Psychological Bulletin, 129, 74-118. Doi: $10.1037 / 0033-2909.129 .1 .74$

Granhag, P. A., \& Hartwig, M. (2015). The Strategic Use of Evidence (SUE) technique: A conceptual overview. In P. A. Granhag, A. Vrij, \& B. Verschuere (Eds.), Deception detection: Current challenges and new approaches (pp. 231-251). Chichester, England: Wiley.

Hartwig, M., Granhag, P. A., \& Strömwall, L. (2007). Guilty and innocent suspects' strategies during interrogations. Psychology, Crime, \& Law, 13, 213-227, doi: $10.1080 / 10683160600750264$.

Jarosz, A. F., \& Wiley, J. (2014). What Are the Odds? A Practical Guide to Computing and Reporting Bayes Factors. The Journal of Problem Solving, 7(1). Doi: 10.7771/1932-6246.1167

Jeffreys, H. (1961). Theory of Probability. Oxford, UK: Oxford University Press. 
Köhnken, G. (2004). Statement Validity Analysis and the 'detection of the truth'. In P. A. Granhag \& L. A. Strömwall (Eds.), Deception detection in forensic contexts (pp. 41-63). Cambridge, England: Cambridge University Press.

Lakens, D. (2016, January 14). Power analysis for default Bayesian $t$-tests [Blog post]. Retrieved from http://daniellakens.blogspot.com/2016/01/poweranalysis-for-default-bayesian-t.html

Leal, S., Vrij, A., Deeb, H., \& Kamermans, K. (2019). Encouraging interviewees to say more and deception: The Ghostwriter method. Legal and Criminological Psychology. Doi:10.1111/lcrp.12152

Leal, S., Vrij, A., Vernham, Z., Dalton, G., Jupe, L., Harvey, A., \& Nahari, G. (2018). Cross-cultural verbal deception. Legal and Criminological Psychology, 23, 192213. Doi: $10.1111 / 1 \mathrm{crp} .12131$

Nahari, G. (2018). The applicability of the Verifiability Approach to the real world. In P. Rosenfeld (Ed.). Detecting concealed information and deception: Verbal, behavioral, and biological methods (pp. 329-350). San Diego, CA: Academic Press. Doi: 10.1016/B978-0-12-812729-2.00014-8.

Nahari, G., Ashkenazi, T., Fisher, R. P., Granhag, P. A., Hershkovitz, I., Masip, J., Meijer, E., Nisin, Z., Sarid, N., Taylor, P. J., Verschuere, B., \& Vrij, A. (2019). Language of Lies: Urgent issues and prospects in verbal lie detection research. Legal and Criminological Psychology, 24, 1-23. Doi:10.1111/lcrp.12148

Nahari, G., Vrij, A., \& Fisher, R. P. (2014). Exploiting liars' verbal strategies by examining the verifiability of details. Legal and Criminological Psychology, 19, 227-239. Doi: 10.1111/j.2044-8333.2012.02069.x 
Sporer, S. L. (2016). Deception and cognitive load: Expanding our horizon with a working memory model. Frontiers in Psychology: Hypothesis and Theory, 7, article 420. Doi 10.3389/fpsyg.2016.00420.

Sporer, S. L., \& Schwandt, B. (2006). Paraverbal indicators of deception: A metaanalytic synthesis. Applied Cognitive Psychology, 20, 421-446. Doi: 10.1002/acp.1190

Sporer, S. L., \& Schwandt, B. (2007). Moderators of nonverbal indicators of deception: A meta-analytic synthesis. Psychology, Public Policy, and Law, 13, 1-34. Doi:10.1037/1076-8971.13.1.1

Taylor, P. J., Larner, S., Conchie, S. M., \& Menacere, T. (2017). Culture moderates changes in linguistic self-presentation and detail provision when deceiving others. Royal Society Open Science, 4, 170128. doi: 10.1098/rsos.170128.

Taylor, P. J., Larner, S., Conchie, S. M., \& van der Zee, S. (2014). Cross-cultural deception detection. In P. A. Granhag, A. Vrij, \& B. Verschuere (Eds.), Detecting deception: Current challenges and cognitive approaches (pp. 175201). Chichester, UK: John Wiley \& Sons.

Vallano, J., P. \& Schreiber Compo, N. (2011). A comfortable witness is a good witness: Rapport-building and susceptibility to mis-information in an investigative mockcrime interview. Applied Cognitive Psychology, 25, 960-970. DOI: 10.1002/acp.1789

Volbert, R., \& Steller, M. (2014). Is this testimony truthful, fabricated, or based on false memory? Credibility assessment 25 years after Steller and Köhnken (1989). European Psychologist, 19, 207-220. doi:10.1027/10169040/a000200. 
Vrij, A. (2008). Detecting lies and deceit: Pitfalls and opportunities, second edition. Chichester: John Wiley and Sons.

Vrij, A., \& Fisher, R. P. (2016). Which lie detection tools are ready for use in the criminal justice system? Journal of Applied Research in Memory and Cognition, 5, 302-307. Doi: 10.1016/j.jarmac.2016.06.014

Vrij, A., Leal, S., \& Fisher, R. P. (2018). Verbal deception and the Model Statement as a lie detection tool. Frontiers in Psychiatry, section Forensic Psychiatry, 9, 492. Doi: $10.3389 /$ fpsyt.2018.00492

Vrij, A., Leal, S., Fisher, R. P., Mann, S., Jo, E., Shaboltas, A., Khaleeva, M., Granskaya, J., \& Houston, K. (2019). Eliciting information and cues to deceit through sketching in interpreter-based interviews. Applied Cognitive Psychology. Doi: 10.1002/acp.3566

Vrij, A., Leal, S., Fisher, R. P., Mann, S., Dalton, G. Jo, E., Shaboltas, A., Khaleeva, M., Granskaya, J., \& Houston, K. (2018a). Sketching as a technique to elicit information and cues to deceit in interpreter-based interviews. Journal of Applied Research in Memory and Cognition, 7, 303-313. Doi:

10.1016/j.jrarmac.2017.11.001

Vrij, A., Leal, S., Jupe, L., \& Harvey, A. (2018b). Within-subjects verbal lie detection measures: A comparison between total detail and proportion of complications. Legal and Criminological Psychology, 23, 265-279. Doi:10.1111/lcrp.12126

Vrij, A., Leal, S., Mann, S., Dalton, G. Jo, E., Shaboltas, A., Khaleeva, M., Granskaya, J., \& Houston, K. (2017). Using the Model Statement to elicit information and cues to deceit in interpreter-based interviews. Acta Psychologica, 177, 44-53. Doi: 10.1016/j.actpsy.2017.04.011 
Vrij, A., Leal, S., Mann, S., Fisher, R. P., Dalton, G. Jo, E., Shaboltas, A., Khaleeva, M., Granskaya, J., \& Houston, K. (2018c). Using unexpected questions to elicit information and cues to deceit in interpreter-based interviews. Applied Cognitive Psychology, 32, 94-104. Doi: 10.1002/acp.3382

Vrij, A. \& Nahari, G. (2019). The Verifiability Approach In J. J. Dickinson, N. Schreiber Compo, R. N. Carol, B. L. Schwartz, \& M. R. McCauley (eds.) Evidence-Based Investigative Interviewing (pp. 116-133). New York, U.S.A.: Routledge Press.

Wetzels, R., \& Wagenmakers, E. J. (2012). A default Bayesian hypothesis test for correlations and partial correlations. Psychonomic Bulletin \& Review, 19, 1057-1064. Doi: 10.3758/s13423-012-0295-x 
Table 1

Statistical Results as a Function of Veracity

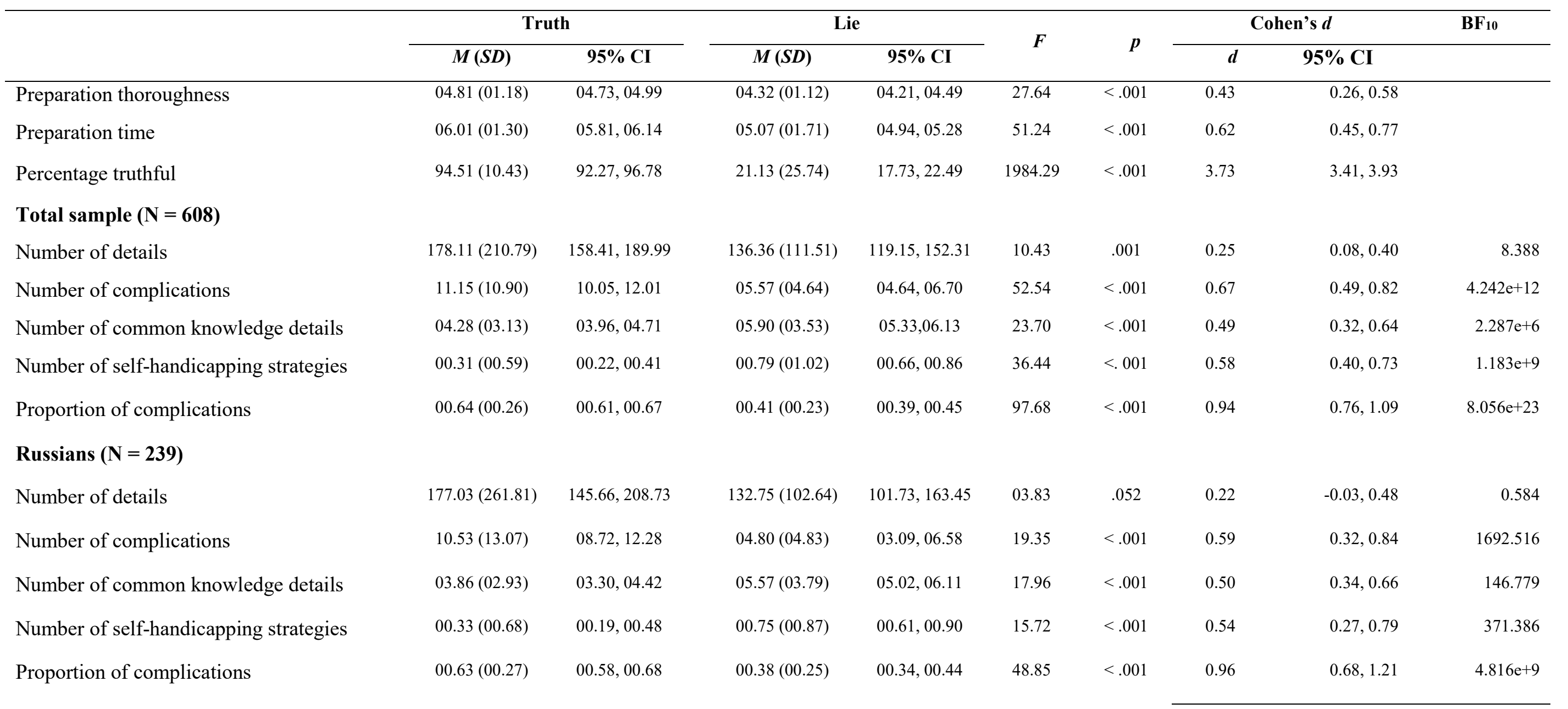




\section{Koreans $(N=240)$}

Number of details

Number of complications

Number of common knowledge details

Number of self-handicapping strategies

Proportion of complications

\section{Hispanics $(\mathrm{N}=129)$}

Number of details

Number of complications

Number of common knowledge details

Number of self-handicapping strategies

Proportion of complications

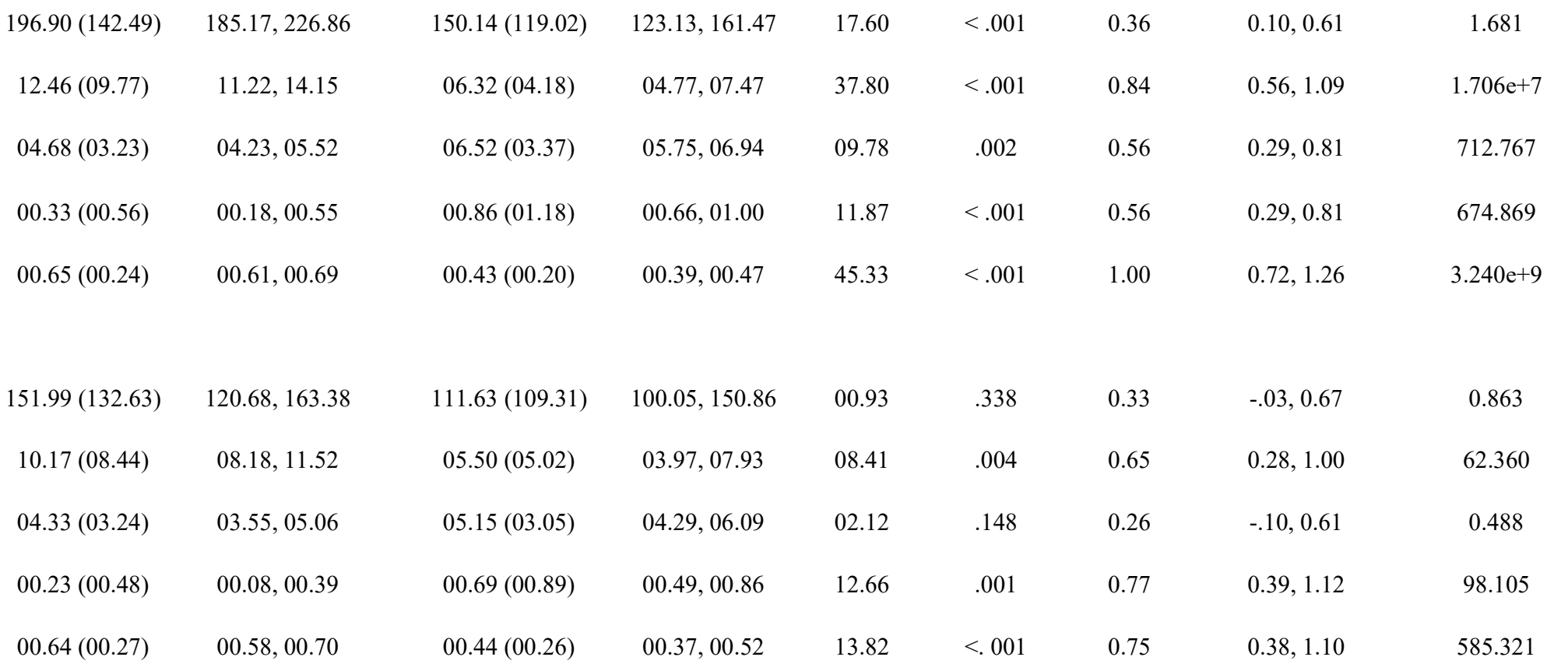


Table 2

Statistical Results as a Function of Nationality

\begin{tabular}{|c|c|c|c|c|c|c|c|c|}
\hline & \multicolumn{2}{|c|}{ Russians } & \multicolumn{2}{|c|}{ Koreans } & \multicolumn{2}{|c|}{ Hispanic } & \multirow[b]{2}{*}{$\boldsymbol{F}$} & \multirow[b]{2}{*}{$p$} \\
\hline & $\mathrm{M}(S D)$ & $95 \% \mathrm{CI}$ & $\mathrm{M}(S D)$ & $95 \% \mathrm{CI}$ & M (SD) & $95 \%$ CI & & \\
\hline Preparation thoroughness & $04.52^{\mathrm{a}}(1.08)$ & {$[03.91,04.08]$} & $04.42^{\mathrm{a}}(01.09)$ & {$[03.82,03.99]$} & $04.91^{\mathrm{b}}(01.39)$ & {$[03.81,4.06]$} & 05.66 & \\
\hline Preparation time & $06.12^{\mathrm{c}}(1.25)$ & {$[04.38,04.66]$} & $04.97^{\mathrm{a}}(01.75)$ & {$[04.30,04.58]$} & $05.53^{\mathrm{b}}(01.47)$ & {$[04.65,5.05]$} & 36.18 & -.00 \\
\hline Rapport & $05.85^{\mathrm{b}}(0.71)$ & {$[05.75,05.95]$} & $05.39^{\mathrm{a}}(00.83)$ & {$[05.30,05.50]$} & $05.28^{\mathrm{a}}(00.86)$ & {$[05.12,05.40]$} & 30.43 & 5.00 \\
\hline Number of details & $154.43(198.13)$ & {$[136.09,172.21]$} & $171.77(158.70)$ & {$[154.97,189.90]$} & $135.09(124.57)$ & {$[114.31,162.31]$} & 02.75 & \\
\hline Complications & $07.61^{\mathrm{a}}(10.17)$ & {$[06.36,08.60]$} & $09.16^{\mathrm{b}}(07.92)$ & {$[08.38,10.55]$} & $08.22^{\mathrm{ab}}(07.55)$ & {$[06.61,09.59]$} & 03.09 & 0 \\
\hline Common knowledge details & $04.73^{\mathrm{a}}(03.49)$ & {$[04.27,05.14]$} & $05.67^{\mathrm{b}}(03.43)$ & {$[05.17,06.01]$} & $04.67^{\mathrm{a}}(03.18)$ & {$[04.23,05.38]$} & 04.63 & .01 \\
\hline Self-handicapping strategies & $00.55(00.81)$ & {$[00.42,00.64]$} & $00.62(00.98)$ & {$[00.49,00.70]$} & $00.42(00.71)$ & {$[00.34,00.64]$} & 00.69 & .50 \\
\hline Proportions of complications & $00.51(00.29)$ & {$[00.47,00.54]$} & $00.53(00.25)$ & {$[00.51,00.58]$} & $00.56(00.28)$ & {$[00.50,00.59]$} & 01.30 & 27 \\
\hline
\end{tabular}

Note. Only mean scores with a different superscript differ significantly from each other $(p<.05)$. 
Table 3.

Accuracy Rates for the Number of Total Details and Complications and the Proportion of Complications as a Function of Veracity.

\begin{tabular}{|c|c|c|c|c|c|c|c|}
\hline & \multicolumn{3}{|c|}{ Hit Rate } & \multirow[t]{2}{*}{$\chi^{2}$} & \multirow[t]{2}{*}{ Wilks' $\lambda$} & \multirow[t]{2}{*}{$p$-value } & \multirow[t]{2}{*}{ Canonical correlation } \\
\hline & $\begin{array}{l}\text { Truths } \\
(\%)\end{array}$ & $\begin{array}{l}\text { Lies } \\
(\%)\end{array}$ & $\begin{array}{l}\text { Total } \\
(\%)\end{array}$ & & & & \\
\hline \multicolumn{8}{|l|}{ Russians } \\
\hline $\begin{array}{l}\text { Number of details } \\
\text { Proportion of complications }\end{array}$ & 65.0 & 72.1 & 68.6 & $\begin{array}{l}2.98 \\
50.20\end{array}$ & $\begin{array}{l}.987 \\
.809\end{array}$ & $\begin{array}{l}.084 \\
<.001\end{array}$ & $\begin{array}{l}.112 \\
.437\end{array}$ \\
\hline \multicolumn{8}{|l|}{ Koreans } \\
\hline Number of details & 36.9 & 69.0 & 54.2 & 5.20 & .978 & .023 & .147 \\
\hline Proportion of complications & 73.0 & 67.4 & 70.0 & 49.39 & .812 & $<.001$ & .433 \\
\hline \multicolumn{8}{|l|}{ Hispanics } \\
\hline $\begin{array}{l}\text { Number of details } \\
\text { Proportion of complications }\end{array}$ & 68.0 & 66.7 & 67.4 & $\begin{array}{l}3.30 \\
17.24\end{array}$ & $\begin{array}{l}.974 \\
.873\end{array}$ & $\begin{array}{l}.069 \\
<.001\end{array}$ & $\begin{array}{l}.160 \\
.357\end{array}$ \\
\hline
\end{tabular}


${ }^{1}$ Vrij et al. (2018c) was a subset of Vrij et al. (2018a).

${ }^{2}$ According to Cohen (1988), effect sizes of $d=20$ are small, effect sizes of $d=.50$ are medium and effect sizes of $d=.80$ are large.

${ }^{3}$ Bayes factors allow for further inferences to be drawn regarding the evidence in an experiment (Jarosz \& Wiley, 2014). Bayes factor analysis is a method to test the probability of the observed data under the null hypothesis compared to the alternative hypothesis (Wetzels \& Wagenmakers, 2012). A BF10 smaller than 1 indicates evidence for the absence of an effect (support of the null hypothesis). According to the cut-off thresholds provided by Jeffreys (1961), BF values between 1 and 3 suggest weak evidence for the alternative hypothesis, values between 3 and 10 suggest substantial evidence, values between 10 and 20 constitute strong evidence, and values above 20 constitute very strong evidence. We used the default Cauchy's prior of .707 for the Bayesian t-tests (Lakens, 2016). 